\title{
Amphetamine-Associated Contextual Learning Is Accompanied by Structural and Functional Plasticity in the Basolateral Amygdala
}

\author{
David J. Rademacher, J. Amiel Rosenkranz, Maud M. Morshedi, Elyse M. Sullivan, and Gloria E. Meredith \\ Department of Cellular and Molecular Pharmacology, Chicago Medical School, Rosalind Franklin University of Medicine and Science, North Chicago, \\ Illinois 60064
}

Drug seeking and the vulnerability to relapse occur when individuals are exposed to an environment with sensory cues in which drug taking has occurred. Memory formation is thought to require plasticity in synaptic circuits, and so we examined whether the memory for a drug-paired environment correlates with changes in the synaptic circuits of the basolateral amygdala (BLA), in which emotional learning is a recognized phenomenon. We used amphetamine (AMPH) as the unconditioned stimulus in the conditioned place preference (CPP) paradigm. Rats were conditioned with $1.0 \mathrm{mg} / \mathrm{kg}$ AMPH and tested, drug free, $72 \mathrm{~h}$ after the last conditioning session. Controls included a saline-conditioned group and a home cage AMPH injection group, whose exposure to the CPP apparatus was delayed by $4 \mathrm{~h}$, long enough to clear the AMPH from the brain. We counted excitatory synapses in the BLA using the electron microscope and the physical disector design (stereology). Rats that expressed AMPH CPP had an increase in excitatory synapses compared with controls. Excitatory synaptic activity was measured using in vivo intracellular recordings from the BLA in anesthetized rats. We found that AMPH CPP, but not drug alone, increased measures of synaptic drive, including the frequency of synaptic events, and the paired-pulse ratio of synaptic inputs to BLA pyramidal neurons. The in vivo findings suggest that the increase in BLA neuronal excitatory drive reflects the change in excitatory synapse number. Thus, context- drug associations are accompanied by structural and functional plasticity in the BLA, findings that have important implications for drug-seeking behavior.

\section{Introduction}

In addiction, exposure to drug-related environments elicits drugseeking behavior. Strong memories of the environment in which drug taking occurred become associated with the rewarding effects of the drug (O'Brien et al., 1998), and current thinking suggests that such associations result in enduring cellular adaptations in the brain (Self and Nestler, 1998). However, little is known about how these associative memories are formed. There are numerous reports that repeated drug administration changes neuronal structures, such as spines and dendrites (see Robinson and Kolb, 2004 for review) and increases the number of excitatory synapses (Morrow et al., 2007; Morshedi et al., 2009), but these data provide little insight into the memory-related component of drug seeking.

It has long been believed that learning rewires circuits, although few studies have linked changes in synaptic connections to memory formation. Long-term potentiation, a cellular correlate of learning and memory, is characterized in the hippocampus

Received Dec. 13, 2009; revised Jan. 21, 2010; accepted Feb. 7, 2010.

This work was funded by Grants DA016662 and DA024790 from the National Institute on Drug Abuse. We thank Figen Seiler of the Electron Microscopy Center at Rosalind Franklin University of Medicine and Science and Karen Goldfarb, Mallika Padival, and Scott Siglin for technical assistance. We also thank Drs. Robert Messing, Kuei Tseng, Anthony West, and Marina Wolf for helpful comments on this manuscript.

Correspondence should be addressed to Dr. Gloria E. Meredith, Rosalind Franklin University of Medicine and Science, 3333 Green Bay Road, North Chicago, IL 60064. E-mail: gloria.meredith@rosalindfranklin.edu.

DOI:10.1523/JNEUROSCI.6165-09.2010

Copyright $\odot 2010$ the authors $\quad 0270-6474 / 10 / 304676-11 \$ 15.00 / 0$ by altered synaptic form but not by a change in the number of synaptic connections (Geinisman et al., 1991, 1993; but see Toni et al., 1999). New synapse formation, however, has been associated with motor skill learning and classical conditioning in the cortex and cerebellum (Black et al., 1990; Kleim et al., 1996, 1997, 2002). Although associative learning can facilitate the strength of inputs examined in vitro (Schroeder and Shinnick-Gallagher, 2005; Stuber et al., 2008; Tye et al., 2008), the brain slice preparation loses much of its synaptic connectivity and natural activity patterns. Few studies have examined such synaptic changes in vivo in mammals, but those that have studied such changes have demonstrated that the strength of excitatory afferents to the basolateral amygdala (BLA), a brain center essential for some forms of affective learning and memory, are facilitated by fear conditioning and cue-specific reward learning (Quirk et al., 1997; Rogan et al., 1997; Tye et al., 2008). However, those investigations did not examine the in vivo synaptic basis for this change. Thus, we do not know whether synaptic changes important for the formation of memories such as those associated with reward exist in vivo and are reflected in naturally occurring synaptic activity.

In the present study, we examined whether context-drug associations lead to changes in synaptic connectivity and enhancements of synaptic strength in the BLA. We used conditioned place preference (CPP), a paradigm in which approach or avoidance behavior is elicited by exposure to an environmental context that has been previously paired with amphetamine (AMPH). Since CPP expression occurs on a drug-free test day, the behavior is 
dependent upon the retrieval of the association between the environment and the affective state produced by the drug (White and Carr, 1985). We found that the association of a context with drug is accompanied by the formation of new excitatory synapses and an increase in the excitatory synaptic drive of BLA neurons. We also found that these synaptic changes did not occur with repeated drug administration alone.

\section{Materials and Methods}

Animals. Male Sprague Dawley rats (Harlan) weighing 200-249 g (49-52 $\mathrm{d}$ old) at the start of the experiment were housed in groups of three. They were handled for $5 \mathrm{~d}$ before behavioral conditioning. Food and water were available ad libitum in the home cages. Rats were maintained on a $12 \mathrm{~h}$ light: dark cycle with lights on at $0700 \mathrm{~h}$. All studies were performed in accordance with the Guide for the Care and Use of Laboratory Animals issued by the National Institutes of Health, and were approved by the Rosalind Franklin University of Medicine and Science Institutional Animal Care and Use Committee.

Drugs. D-Amphetamine sulfate (Sigma) was dissolved in sterile $0.9 \%$ saline (vehicle) and injected at a volume of $1 \mathrm{ml} / \mathrm{kg}$, i.p. Doses refer to the drug base. DNDS (4,4'-dinitrostilbene-2,2' -disulfonate, $500 \mu \mathrm{M}$ ) (Tocris Cookson) was dissolved in $2 \mathrm{M}$ potassium acetate and was included in the recording electrode.

Conditioned place preference. During the pretest, each rat was placed into the center chamber $72 \mathrm{~h}$ before conditioning and allowed free access to the entire box. Three of 47 rats $(6 \%)$ that spent $>70 \%$ of their time in either of the two outer chambers were excluded from the experiment. Rats remaining in the experiment were randomly assigned to one of three treatments. In the experimental group $(n=16)$, AMPH $(1.0 \mathrm{mg} / \mathrm{kg}$, i.p. $)$ was administered to each rat on days 1,3 , and 5 immediately before being placed into an outer chamber. On days 2 and 4 , each rat was injected with sterile saline $(1 \mathrm{ml} / \mathrm{kg}$, i.p.) and immediately confined to the opposite outer chamber. Thus, for these rats, the rewarding effect of AMPH was exclusively and reliably paired with one distinct context and the effect of saline was exclusively and reliably paired with another distinct context. The experience of these animals was predicted to produce a robust association between the rewarding effect of AMPH and one distinct environment and weak associations between other emotion-provoking stimuli and the home cage context. We controlled for the effects of drug exposure with a delayed-pairing group $(n=14)$. Each rat in the delayedpairing group was subjected to the identical conditioning schedule with AMPH $(1.0 \mathrm{mg} / \mathrm{kg})$, but remained in his home cage for $4 \mathrm{~h}$ before being placed into an outer chamber for $45 \mathrm{~min}$. Four h was selected for the delay, since this time lag is sufficient to ensure that AMPH has cleared the brain (Honecker and Coper, 1975). Each rat in the final group received sterile saline injections $(n=14)$ before being placed into an outer chamber on days 1,3, and 5 and into the other outer chamber on days 2 and 4 . Conditioning occurred over a 5-day period and each session lasted for 45 min. Between conditioning sessions, the floors and walls of the apparatus were thoroughly washed and dried before being replaced.

Approximately $72 \mathrm{~h}$ after the last conditioning session, all rats were drug- or saline-free, and tested for their preference by being placed into the central chamber followed by the removal of the dividing partitions. The rat was then allowed to explore the entire apparatus for $30 \mathrm{~min}$. Time spent in each compartment was recorded and place preference (i.e., time spent in the paired chamber minus time spent in the unpaired chamber during the CPP test) and locomotion were calculated.

Electron microscopy and stereology. Procedures to prepare brain tissue for electron microscopy were modified from a previous study (Morshedi et al., 2009). Either 1 or $24 \mathrm{~h}$ after behavioral conditioning, rats were deeply anesthetized with sodium pentobarbital (100 mg/kg, i.p.) and transcardially perfused with $10 \mathrm{ml}$ of $0.1 \mathrm{M}$ PBS, followed by $50 \mathrm{ml}$ of $3.75 \%$ acrolein in buffered $2 \%$ paraformaldehyde (PF), pH 7.4 and 200 $\mathrm{ml}$ of buffered $2 \% \mathrm{PF}$. All solutions were made at the same time and the volume of each perfusate was kept constant. The brains were removed, postfixed for $30 \mathrm{~min}$ in buffered $2 \% \mathrm{PF}$, blocked including the BLA (relative to the bregma, anterior-posterior, $-1.8 \mathrm{~mm}$ to $-4.8 \mathrm{~mm}$ ) and then cut coronally $(60 \mu \mathrm{m})$ on a Vibratome (Vibratome). All sections were collected serially in $0.1 \mathrm{~m}$ phosphate buffer into 24 -well culture plates.

The analysis involved an unbiased approach to estimate the total number of asymmetric synapses (physical disector) (Fig. 1). After a random start, every fifth section was selected, yielding a series of 10 equally spaced sections. A second series of 10 equally spaced, adjacent sections were collected to estimate the total volume of the BLA $\left(V_{\text {ref }}\right)$ using the Cavalieri method. The sections selected for the synaptic (electron microscopic) analysis were fixed in $1 \%$ osmium tetroxide (diluted in $0.1 \mathrm{M}$ phosphate buffer) for $30 \mathrm{~min}$ at room temperature in the dark, dehydrated, and flat embedded in epoxy resin 812 (EMbed 812, nadic methyl anhydride, dodecenyl succinic anhydride, benzyldimethylamine; Electron Microscopy Sciences) between two sheets of aclar fluorohalocarbon film. The epoxy resin was allowed to polymerize at $60^{\circ} \mathrm{C}$ for $72 \mathrm{~h}$. An area of known size $(0.5 \times 0.5 \mathrm{~mm})$ was selected from the sections, chosen by systematic random sampling (Calverley et al., 1988). Ultrathin serial sections (silver, $\sim 60-70 \mathrm{~nm}$ ) were cut $(\sim 10$ per grid; EM UC6; Leica), mounted on formvar-coated copper slot grids, and stained with uranyl acetate and lead citrate before viewing. All electron microscopic images were captured on a JEOL JEM-1230 electron microscope equipped with a Hamamatsu ORCA HR CCD camera. A disector analysis was performed on serial ultrathin sections from each block (Fig. 1). Using a systematic sampling method, whereby micrographs are taken at least two widths of the electron microscope screen apart, 15 images of the same region in four adjacent ultrathin sections of the same thickness were taken and used as the reference and look-up sections (Hunter and Stewart, 1993; Ingham et al., 1998; Day et al., 2006). We used 180 disectors for each animal per treatment group (total of 900 disectors per group). Asymmetric synapses and their associated postsynaptic targets were identified using well established criteria (Peters et al., 1991). A rectangular, unbiased counting frame $\left(32.02 \mu \mathrm{m}^{2}\right)$ containing two inclusion and two exclusion lines was placed over each of the micrographs (Fig. 1). Synapses that were within the counting frame or on the inclusion but not the exclusion lines were counted in the reference section only if the same synapse was not found in the look-up section (referred to as "tops"). Sections were then reversed so that the look-up section served as the reference section (Fig. 1). Estimation of mean synaptic density $\left(N_{\mathrm{v} \text { syn }}\right)$ (synapse number per $\mu \mathrm{m}^{3}$ ) was calculated using the formula $N_{\mathrm{v} \text { syn }}=$ $\sum Q_{-}{ }_{\text {syn }} /(h \times A)$, adapted from Sterio (1984) and de Groot and Bierman (1986), where $Q_{-{ }_{\text {syn }}}$ is the number of asymmetric synapses present in the reference section but not the look-up section (tops), $h$ is the distance (micrometers) between disector planes (section thickness), and $A$ is the sample area (square micrometers). Finally, two folds from each ultramicrotome section used for analysis were photographed. Section thickness (h) was estimated as half the mean width of the measured folds (De Groot, 1988). To estimate the absolute number of synapses $(N)$ onto dendritic structures, the following formula was used: $N=N_{\mathrm{v} \text { syn }} \times V_{\text {ref- }}$. For physical disectors, estimate precision was expressed as the coefficient of error for the group. The adjacent series of 10 equally spaced sections were mounted onto poly-L-lysine-coated slides from distilled water, dried at $60^{\circ} \mathrm{C}$ overnight, and then stained with cresyl violet. The reference volume $\left(V_{\text {ref }}\right)$ was estimated using a Nikon E400 microscope equipped with a stage motorized in three axes and a video camera linked to a computer running StereoInvestigator (Microbrightfield) software. To estimate the total volume of the BLA ( $V_{\text {ref }}$, expressed in cubic micrometers), the following formula was used: $V_{\text {ref }}=a \times t \times s$, where $a$ is mean area (square micrometers) of the BLA, $t$ is mean thickness (micrometers) of the Vibratome sections, and $s$ is the total number of sections through the BLA $(\sim 50)$.

In vivo intracellular electrophysiology. Rats from the three groups (AMPH CPP $n=7$; delayed pairing $n=5$; saline conditioned $n=5$ ) were anesthetized $(400 \mathrm{mg} / \mathrm{kg}$ chloral hydrate) and placed in a stereotaxic device (David Kopf Instruments). Supplemental anesthesia (8\% chloral hydrate) was administered as needed, and determined by monitoring the hindlimb withdrawal reflex and cortical EEG. Core body temperature was maintained at $37^{\circ} \mathrm{C}$. Burr holes were drilled, and the dura mater overlying the BLA (5.0 mm lateral, $3.3 \mathrm{~mm}$ caudal from the bregma) was removed. A concentric bipolar stimulation electrode was lowered to the entorhinal/subiculum area. Glass electrodes were filled with $1-2 \%$ neu- 
robiotin in $2 \mathrm{M}$ potassium acetate. In some experiments, DNDS $(500 \mu \mathrm{M})$ was added to the electrode to block chloride/GABA channels (Bridges et al., 1989; Dudek and Friedlander, 1996). Electrodes were lowered slowly to the BLA via a hydraulic micromanipulator (David Kopf Instruments). Voltage signals were amplified (IR-183 intracellular amplifier, Cygnus Technology) and monitored by an oscilloscope (B-K Precision Instruments) and an audio amplifier (AM-10, Grass Technologies). Data were also fed from the amplifier to a personal computer (Mac Pro, Apple), monitored online using Axograph X software (Axograph Scientific) and stored on a hard disk for analysis offline. A stimulator (S88 stimulator, Grass Technologies) in series with the amplifier and a stimulus isolation unit was used to deliver DC to the recording electrode. To confirm the location of recorded neurons, neurobiotin was iontophoresed into the recorded neuron for $2-10 \mathrm{~min}$. After the conclusion of experiments, rats were perfused with isotonic saline followed by ice-cold $4 \% \mathrm{PF}$ in $0.1 \mathrm{M}$ phosphate buffer. After overnight postfixation and cryoprotection in $15 \%$ sucrose buffer, frozen sections $(60-80 \mu \mathrm{m})$ were cut through the BLA. Standard ABC reactions (Elite kit, Vector Immunochemicals) were used to stain filled neurons in BLA sections. All sections were counterstained with cresyl violet to localize the stimulation and recording sites, verified by the position of the filled neuron or the electrode tract. Neurons found to lie outside the BLA were excluded from analysis.

Upon impalement, neurons were given at least $5 \mathrm{~min}$ to stabilize before data were recorded. The resting membrane potential was obtained. Input resistance was derived from the slope of the linear fit of the voltage responses to current injections $(-100$ to +25 $\mathrm{pA}, 800 \mathrm{~ms})$. The peak amplitude from rest and frequency of spontaneously occurring synaptic potentials were quantified. Because spontaneous synaptic events often overlap, we also measured the SD from the mean membrane potential as an unbiased quantification that reflects the amplitude and frequency of spontaneous events (Rosenkranz and Grace, 2002). The amplitudes of EPSPs evoked by electrical stimulation were also measured $(0.1 \mathrm{~ms}$ duration, $0.2 \mathrm{~Hz}, 0.1-1.0 \mathrm{~mA}$ ). Only evoked responses that displayed features consistent with monosynaptic EPSPs were analyzed (monotonic rising phase on visual inspection). For analysis of paired-pulse ratio and coefficient of variance $(\mathrm{CV})$ of EPSP amplitude, a stimulation intensity that evoked EPSPs of $5-10 \mathrm{mV}$ was used. Paired pulses had an interval of $50 \mathrm{~ms}$; short enough to allow functional interaction between pulses but long enough to allow clear discrimination of evoked EPSPs in bridge mode (non-voltage clamp). Neurons that displayed very low input resistance $(<15 \mathrm{M} \Omega)$, a depolarized membrane potential $(<-60 \mathrm{mV})$, or action potentials that did not overshoot $0 \mathrm{mV}$ were excluded from the analysis. Neurons were considered BLA pyramidal neurons if they were histologically confirmed to lie within the BLA, displayed electrophysiological characteristics consistent with BLA pyramidal neurons in vivo, as described previously (Lang and Paré, 1997; Rosenkranz and Grace, 2002, 2003) and, after staining for neurobiotin, were found to be of pyramidal morphology (McDonald, 1982; Millhouse and DeOlmos, 1983).

b
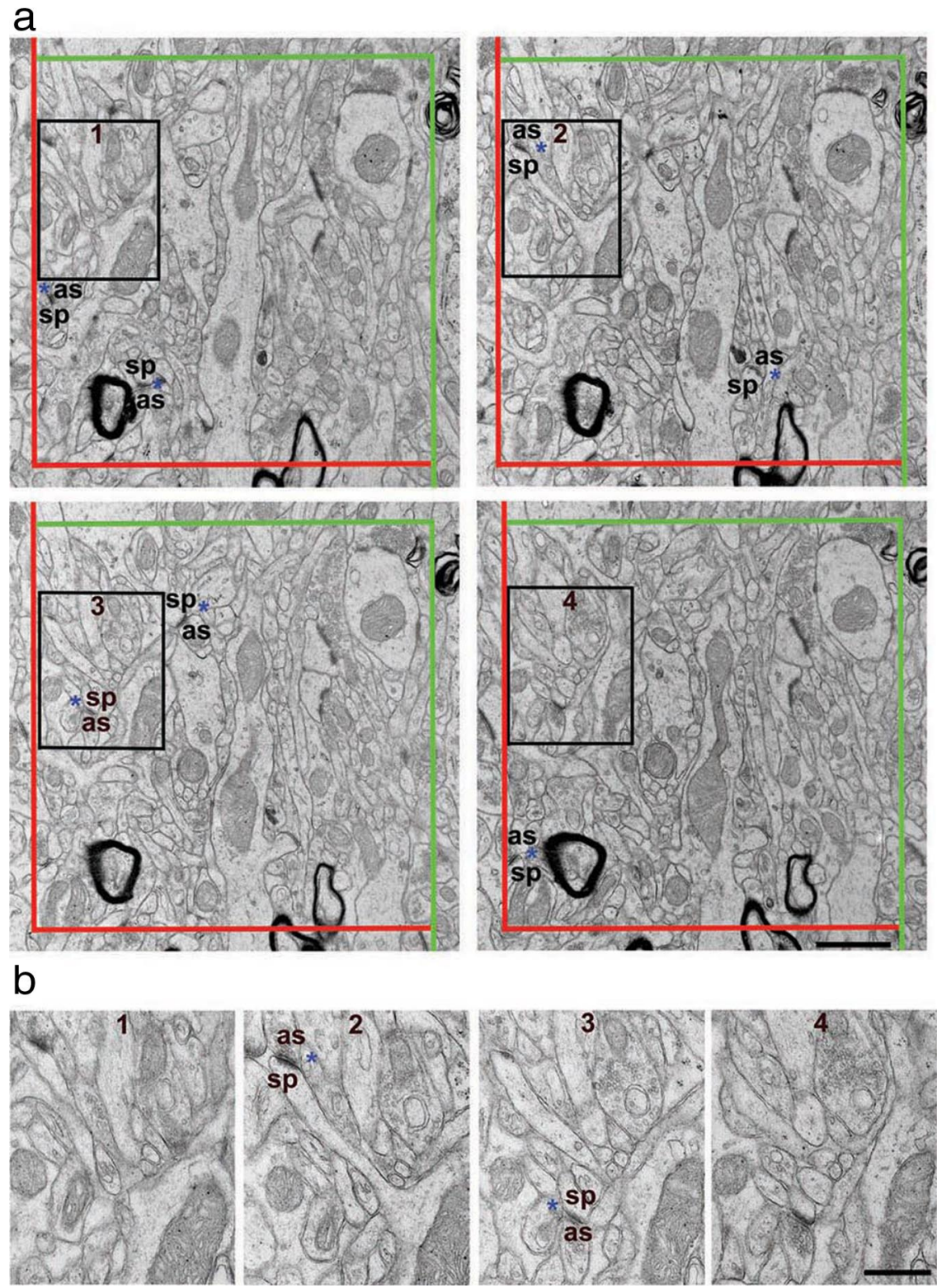

Figure 1. Evaluating synapses using unbiased stereology and electron microscopy (physical disector). $\boldsymbol{a}$, Set of four electron micrographs overlaid with counting frames and taken from corresponding areas of four ultramicrotome serial sections in the BLA. Blue asterisks mark the tops of synapses. Tops were counted if they were within the counting frame or on the inclusion (green lines) but not the exclusion lines (red lines). Each micrograph was used as the reference and the look-up section with its matching pair. Tops of synapses are labeled according to their membrane specialization [asymmetric (as)] and their postsynaptic target [spine (sp)]. The scale bar in the lower right panel equals $1 \mu \mathrm{m}$ and is valid for all four panels. $\boldsymbol{b}$, High-power view of the area within the black box drawn on the left side of each of four sections in $\boldsymbol{a}$. In panel 2 , an asymmetric axospinous synapse is considered a top of a synapse because a synaptic cleft, postsynaptic density, and neurotransmitter vesicles are apparent in panel 2 but not 1 . Similarly, in panel 3 , an asymmetric axospinous synapse is considered a top of a synapse because a synaptic cleft, postsynaptic density, and neurotransmitter vesicles are apparent in panel 3 but not 2. Scale bar: panel 4 (for panels 1-4), $500 \mathrm{~nm}$.

Statistical analysis. Synaptic data were analyzed with two-way ANOVA with Bonferroni post-tests. The linear relationships between synaptic and electrophysiological variables and CPP scores were determined by calculating the Pearson correlation coefficient $(r)$. Electrophysiological and CPP data were analyzed with one-way ANOVA with post hoc Student-Newman-Keuls tests. Before statistical analysis, normality of distribution was determined with Kolmogorov-Smirnov test; equality of variance was tested using Barlett's test. The $\alpha$ level was set at 0.05 . A Bonferroni correction was used to reduce the $\alpha$ level when multiple post hoc comparisons were made. The reduction in the $\alpha$ level depended upon the number of post hoc comparisons. All data are represented as mean \pm SEM. In instances when more than one neuron was recorded electro- 


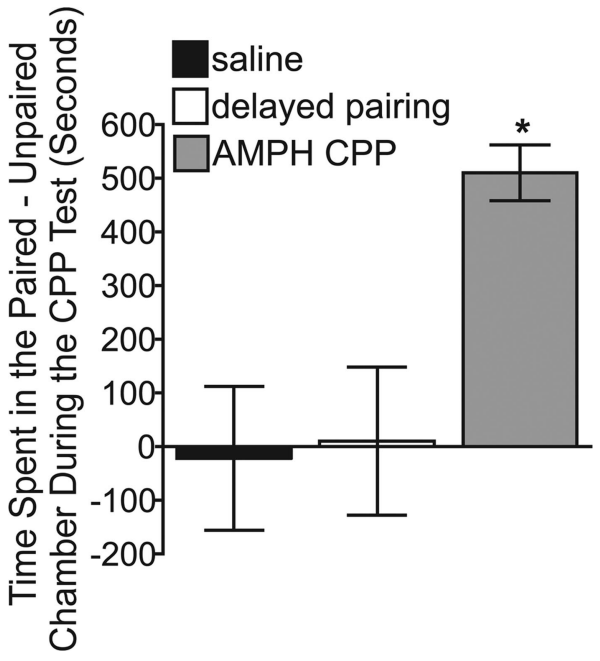

Figure 2. Behavioral conditioning with AMPH produced AMPH CPP. Animals treated with AMPH and immediately paired with a specific chamber spent more time in the AMPH-paired than the unpaired chamber during the CPP test. Animals treated with the delayed-pairing regimen or with saline alone did not exhibit a preference for one chamber over the other during the CPP test. Data are expressed as the mean \pm SEM. ${ }^{*} p<0.01$ versus the delayed-pairing and saline groups.

physiologically from one rat, for the primary analysis, each neuron was analyzed as an individual data point in the dataset (with $n$ equal to the number of neurons). Whenever the results from this analysis provided statistically significant outcomes, we also took a secondary conservative approach, averaging the values of all neurons obtained from a single rat and running analyses on these collapsed values. Where applicable, the $p$ values for these analyses are presented after the primary analysis and noted as $p_{a}$ (probability for analyses based on animal number instead of neuron number).

\section{Results}

\section{Repeated pairings of AMPH with context produces AMPH CPP}

We conditioned two groups of rats with AMPH and saline, or saline alone, and exposed them to chamber-specific cues paired to treatment (see Materials and Methods). Rats were tested for chamber preference when drug free and their brains prepared for neuroanatomical studies at 1 or $24 \mathrm{~h}$ after the test. Rats used in the electrophysiology studies were prepared for study between 12-24 $\mathrm{h}$ after the test (see Materials and Methods). The timing of the electrophysiological studies varied and was based on the anatomical determination of excitatory synaptic changes following the CPP test. The separate group of rats that controlled for drug exposure was the delayed-pairing group. As expected from our previous work (Rademacher et al., 2006; Shen et al., 2006), we found that neither AMPH treatment with the delayed-pairing regimen nor treatment with saline alone resulted in a preference for one compartment over the other (Fig. 2) ( $p>0.05$ ). In contrast, animals treated with AMPH and immediately paired with a specific chamber spent significantly more time in the AMPHpaired than the unpaired chamber during the CPP test (Fig. 2) (time spent in the drug-paired minus the unpaired context; one-way ANOVA, $F_{(2,41)}=7.89 ; p<0.01$; AMPH CPP versus saline $p<0.01$; AMPH CPP versus delayed pairing $p<0.01$ ). Thus, the increase in time spent in the AMPH-paired chamber was due to a context-drug association rather than repeated exposure to AMPH.

\section{The number of synapses increase with AMPH CPP}

We quantified the excitatory synapses in the BLA of each group at 1 and $24 \mathrm{~h}$ after the CPP test with the physical disector design (stereology) at the electron microscopic level. We found no difference in the total BLA volume between the AMPH CPP, delayed-pairing, and saline groups at 1 and $24 \mathrm{~h}$ after the CPP test [AMPH CPP $(1 \mathrm{~h}) 2.17 \pm 0.03 \mathrm{~mm}^{3}, n=4$ rats; AMPH CPP $(24 \mathrm{~h}) 2.15 \pm 0.04 \mathrm{~mm}^{3}, n=5$ rats; delayed pairing $(1 \mathrm{~h}) 2.20 \pm$ $0.05 \mathrm{~mm}^{3}, n=4$ rats; delayed pairing $(24 \mathrm{~h}) 2.18 \pm 0.05 \mathrm{~mm}^{3}$, $n=5$ rats; saline $(1 \mathrm{~h}) 2.18 \pm 0.05 \mathrm{~mm}^{3}, n=4$ rats; saline $(24 \mathrm{~h})$ $2.15 \pm 0.07 \mathrm{~mm}^{3}, n=5$ rats; two-way ANOVA, no main effect of behavioral conditioning (AMPH CPP, delayed pairing, saline), $F_{(2,21)}=0.20 ; p>0.05$, no main effect of time after the CPP test $\left.(1 \mathrm{~h}, 24 \mathrm{~h}) F_{(1,21)}=0.44 ; p>0.05\right]$. We estimated from vehicle-treated rats that the BLA contains $3.30 \pm 0.21 \times 10^{9}$ asymmetric, presumed excitatory, synapses, $\sim 97 \%$ of which were single synaptic boutons, with the remainder being multisynaptic boutons (MSBs) (Fig. 3a,b). When separated by synaptic target, axospinous synapses make up the vast majority $(88 \%)$ of excitatory contacts; axodendritic terminals comprise $10 \%$ of these contacts; and the remaining synapses (2\%) could not be classified for technical reasons (see Materials and Methods) (Fig. 3a,b).

\section{Specificity of synaptic changes}

It was important to distinguish synaptic modifications associated with contextual learning from those that might result directly from drug exposure, since repeated AMPH administration without conditioning increases the number of excitatory synapses (quantified by using unbiased stereology) in the prefrontal cortex (Morrow et al., 2007; Morshedi et al., 2009). It was also important to determine whether the synaptic changes were due to a contextdrug association or exposure to the CPP apparatus during the $\mathrm{CPP}$ test. We found that repeated drug exposure (delayed-pairing group) did not change the total number or density of asymmetric (excitatory) synapses in the BLA compared with the salinetreated rats (Fig. $3 c$, Table 1) [number $(1 \mathrm{~h}) p>0.05$; number $(24 \mathrm{~h}) p>0.05$; density $(1 \mathrm{~h}) p>0.05$; density $(24 \mathrm{~h}) p>0.05$ ]. However, the AMPH CPP group did show a significant increase in the total number of excitatory synapses compared with controls (Fig. 3c) [two-way ANOVA, main effect of behavioral conditioning, $F_{(2,21)}=30.51 ; p<0.0001$; AMPH CPP versus saline $(1 \mathrm{~h}) p<0.001$; AMPH CPP versus saline $(24 \mathrm{~h}) p<0.001$; AMPH CPP versus delayed pairing $(1 \mathrm{~h}) p<0.001$; AMPH CPP versus delayed pairing $(24 \mathrm{~h}) p<0.01]$. Importantly, there was no difference in the total number of asymmetric synapses at $1 \mathrm{~h}$ compared with $24 \mathrm{~h}$ after the CPP test (Fig. 3c) ( $p>0.05)$, data consistent with the notion that increases in the number of synapses resulted from the earlier formation of context-drug memories rather than the recent recall or reconsolidation of the memory. Because the volume remained constant between the groups, the density of excitatory synapses was increased in the AMPH CPP group compared with controls at both 1 and $24 \mathrm{~h}$ after the CPP test (Table 1) [two-way ANOVA, main effect of behavioral conditioning $F_{(2,21)}=25.22 ; p<0.0001$; AMPH CPP versus saline (1 h) $p<0.001$; AMPH CPP versus saline $(24 \mathrm{~h}) p<0.001$; AMPH CPP versus delayed pairing $(1 \mathrm{~h}) p<0.01$; AMPH CPP versus delayed pairing $(24 \mathrm{~h}) p<0.01]$. There was no difference in the density of asymmetric synapses at $1 \mathrm{~h}$ compared with $24 \mathrm{~h}$ after the CPP test (Table 1) $(p>0.05)$.

We also related the number of excitatory synapses to their synaptic target (spines or dendrites). Again, we found no difference between the saline and delayed-pairing groups in the number or density of axospinous (Fig. 3c, Table 1) [number (1 h) $p>0.05$; 

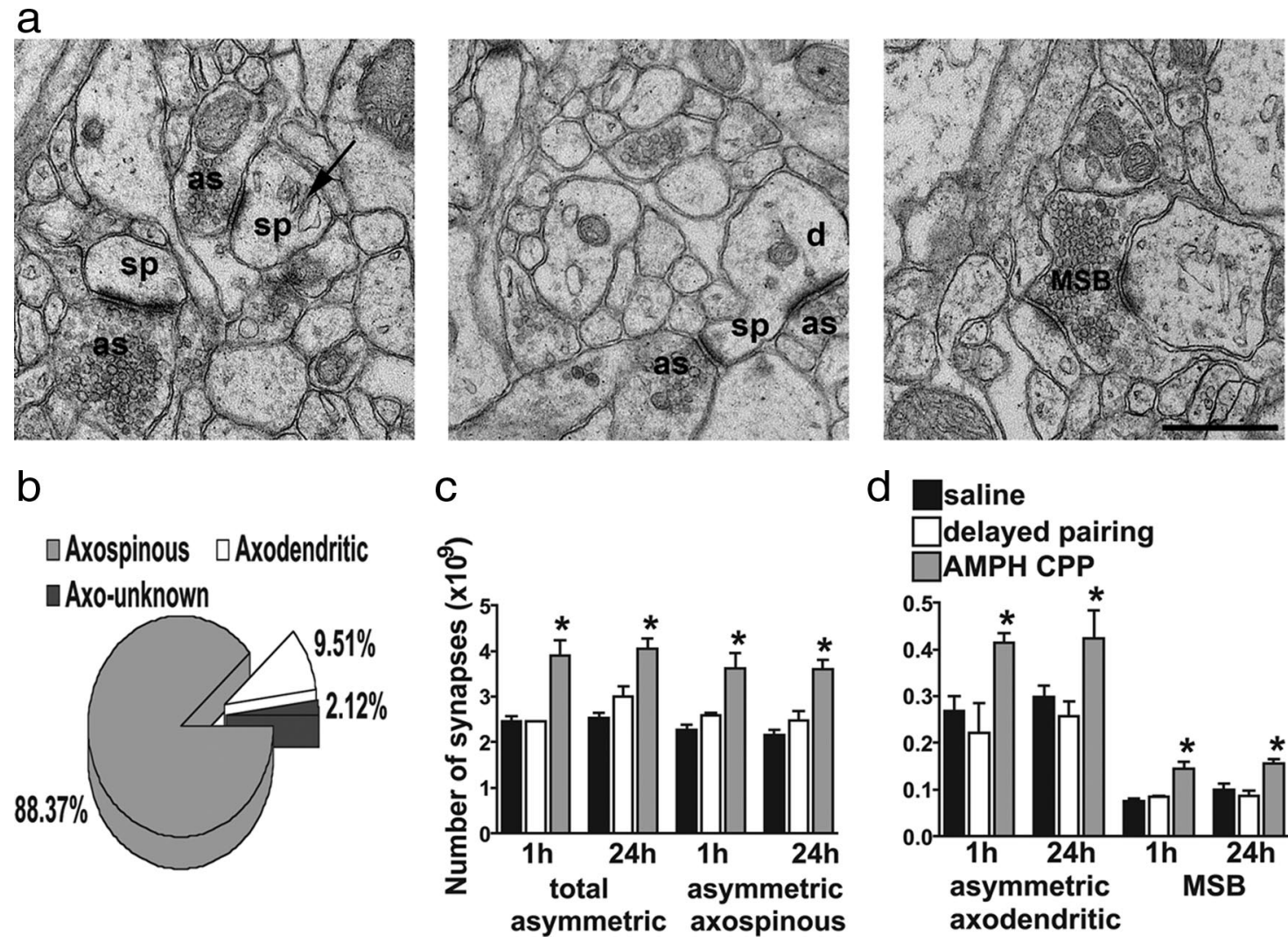
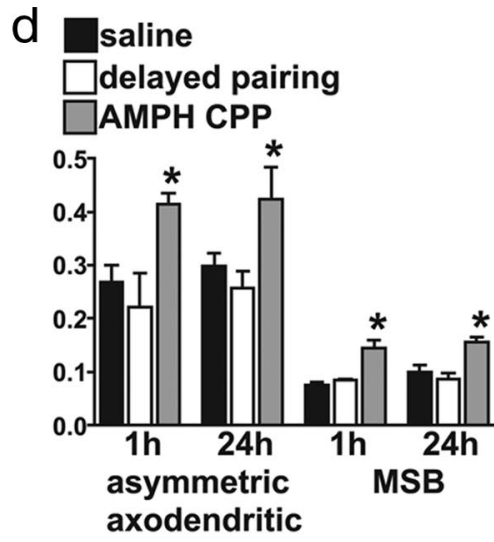

Figure 3. AMPH CPP produced structural indices of increased excitatory input. $\boldsymbol{a}$, Electron micrographs demonstrating asymmetric, presumably excitatory, synapses in the BLA. In the left panel, the arrow points to a spine apparatus. In the middle panel, an asymmetric synapse on a spine head and an asymmetric synapse on the shaft of the dendrite are marked. In the right panel, an electron micrograph depicts a multisynaptic bouton in the BLA. as, Asymmetric specialization; sp, spine; $d$, dendrite. The scale bar equals $500 \mathrm{~nm}$ and is valid for all three panels. $\boldsymbol{b}$, Pie chart illustrating the percentage of single synaptic boutons classified as axospinous, axodendritic, or synapses onto unknown postsynaptic targets (axo-unknown). $c$, The total number of asymmetric synapses and the number of asymmetric axospinous synapses were greater for rats in the AMPH CPP group at 1 and $24 \mathrm{~h}$ after the CPP test compared with animals in the delayed-pairing and saline groups. There were no differences in the total number of asymmetric synapses and the number of asymmetric axospinous synapses for rats in the delayed-pairing group compared with the saline group at either 1 or $24 \mathrm{~h}$ after the CPP test. Data are expressed as the mean \pm SEM. $\boldsymbol{d}$, The number of asymmetric axodendritic synapses and MSBs was greater for animals in the AMPH CPP group at 1 and $24 \mathrm{~h}$ after the CPP test compared with animals in the delayed-pairing and saline groups. There was no difference in the number of asymmetric axodendritic synapses and MSBs for rats in the delayed-pairing group compared with the saline group at either 1 or $24 \mathrm{~h}$ after the CPP test. Data are expressed as the mean \pm SEM. ${ }^{*} p<0.05$ versus the delayed-pairing and saline groups.

Table 1. The density of excitatory synapses in the BLA for each group measured at 1 and $24 \mathrm{~h}$ after the CPP test

\begin{tabular}{llll}
\hline \multirow{2}{*}{ Synapse type } & \multicolumn{3}{l}{ Behavioral conditioning regimen } \\
\cline { 2 - 4 } & Saline & Delayed pairing & AMPH CPP \\
\hline Total asymmetric $(1 \mathrm{~h})$ & $1.08 \pm 0.06$ & $1.28 \pm 0.01$ & $1.77 \pm 0.19^{*}$ \\
Total asymmetric $(24 \mathrm{~h})$ & $1.18 \pm 0.06$ & $1.37 \pm 0.11$ & $1.89 \pm 0.10^{*}$ \\
Asymmetric axospinous $(1 \mathrm{~h})$ & $1.00 \pm 0.07$ & $1.17 \pm 0.04$ & $1.64 \pm 0.21^{*}$ \\
Asymmetric axospinous $(24 \mathrm{~h})$ & $1.01 \pm 0.05$ & $1.14 \pm 0.09$ & $1.68 \pm 0.09^{*}$ \\
Asymmetric axodendritic $(1 \mathrm{~h})$ & $0.12 \pm 0.02$ & $0.10 \pm 0.02$ & $0.19 \pm 0.01^{*}$ \\
Asymmetric axodendritic $(24 \mathrm{~h})$ & $0.14 \pm 0.01$ & $0.12 \pm 0.01$ & $0.20 \pm 0.02^{*}$ \\
Multisynaptic boutons $(1 \mathrm{~h})$ & $0.03 \pm 0.004$ & $0.04 \pm 0.002$ & $0.07 \pm 0.011^{*}$ \\
Multisynaptic boutons $(24 \mathrm{~h})$ & $0.05 \pm 0.006$ & $0.04 \pm 0.005$ & $0.07 \pm 0.004^{*}$ \\
\hline
\end{tabular}

Data are expressed as mean synapses per cubic micrometer \pm SEM. ${ }^{*} p<0.05$ versus delayed pairing and saline groups.

number $(24 \mathrm{~h}) p>0.05$; density $(1 \mathrm{~h}) p>0.05$; density $(24 \mathrm{~h}) p>$ 0.05 ] or axodendritic synapses (Fig. $3 d$, Table 1$)$ [number (1 h) $p>0.05$; number $(24 \mathrm{~h}) p>0.05$; density $(1 \mathrm{~h}) p>0.05$; density (24 h) $p>0.05]$. In contrast, the total number of asymmetric axospinous and axodendritic synapses was significantly increased in the AMPH CPP group compared with delayed pairing and saline controls at 1 and $24 \mathrm{~h}$ after the CPP test (Fig. $3 c, d$ ) [asymmetric axospinous synapses: two-way ANOVA, main effect of behavioral conditioning $F_{(2,21)}=29.49$; $p<0.0001$; AMPH $\mathrm{CPP}$ versus saline $(1 \mathrm{~h}) p<0.001$; AMPH CPP versus saline
(24 h) $p<0.001$; AMPH CPP versus delayed pairing $(1 \mathrm{~h}) p<0.01$; AMPH CPP versus delayed pairing $(24 \mathrm{~h}) p<0.001$; asymmetric axodendritic synapses: two-way ANOVA, main effect of behavioral conditioning $F_{(2,21)}=9.53$, $p<0.01$; AMPH CPP versus saline $(1 \mathrm{~h}) p<0.05$; AMPH CPP versus saline $(24 \mathrm{~h}) p<0.05$; AMPH CPP versus delayed pairing $(1 \mathrm{~h}) p<0.05$; AMPH CPP versus delayed pairing $(24 \mathrm{~h}) p<0.05]$. There was no difference in the number of asymmetric axospinous (Fig. $3 c)(p>0.05)$ or axodendritic (Fig. $3 d)(p>0.05)$ synapses at $1 \mathrm{~h}$ compared with $24 \mathrm{~h}$ after the CPP test (Table 1$)$.

We can therefore conclude that the repeated association of context with drug rather than the effects of drug alone or exposure to the CPP apparatus during the test were responsible for the increase in the density of asymmetric axospinous (Table 1) [twoway ANOVA, main effect of behavioral conditioning $F_{(2,21)}=$ 22.94; $p<0.0001$; AMPH CPP versus saline (1 h) $p<0.001$; AMPH CPP versus saline $(24 \mathrm{~h}) p<0.001$; AMPH CPP versus delayed pairing $(1 \mathrm{~h}) p<0.05$; AMPH CPP versus delayed pairing (24 h) $p<0.01$ ] and axodendritic (Table 1) [two-way ANOVA main effect of behavioral conditioning $F_{(2,21)}=16.17 ; p<$ 0.0001; AMPH CPP versus saline $(1 \mathrm{~h}) p<0.05$; AMPH CPP versus saline $(24 \mathrm{~h}) p<0.05$; AMPH CPP versus delayed pairing ( $1 \mathrm{~h}) p<0.01$; AMPH CPP versus delayed pairing $(24 \mathrm{~h}) p<$ 0.05 ] synapses observed in the AMPH CPP group compared with 
Table 2. Linear relationship between the CPP score (i.e., time spent in the AMPHpaired minus the time spent in the unpaired chamber during the (PP test) and synaptic parameters evaluated at $24 \mathrm{~h}$ after the CPP test in the BLA

\begin{tabular}{llll}
\hline & \multicolumn{3}{c}{ CPP score and } \\
\cline { 2 - 4 } & Synapses (number) & Synapses (density) & Synaptic events \\
\hline Asymmetric & $0.47^{*}$ & $0.46^{*}$ & \\
Asymmetric axospinous & $0.47^{*}$ & $0.47^{*}$ & \\
Asymmetric axodendritic & 0.14 & 0.13 & \\
Multisynaptic boutons & $0.61^{*}$ & $0.61^{*}$ & $0.48^{*}$ \\
Spontaneous synaptic events & & & \\
\hline
\end{tabular}

Data reveal Pearson's $r,{ }^{*} p<0.05$

delayed pairing and saline control groups. There was no difference in the number of asymmetric axospinous $(p>0.05)$ or axodendritic $(p>0.05)$ synapses at $1 \mathrm{~h}$ compared with $24 \mathrm{~h}$ after the CPP test. Thus, animals that associated the environmental context with AMPH have an increased number of excitatory inputs onto BLA neurons, and especially onto pyramidal cells, since they receive the bulk of the excitatory inputs onto their spines (Brinley-Reed et al., 1995).

The observation that excitatory synapses onto pyramidal neurons increase after AMPH CPP raised the possibility that synaptic connections had been redistributed or remodeled (Knott et al., 2006). Our data are not consistent with a redistribution, since we found no difference in the proportion of single to multiple synaptic boutons, or of axospinous, axodendritic, and unclassified synapses between groups at 1 or $24 \mathrm{~h}$ after the CPP test (two-way ANOVA, no main effect of behavioral conditioning $F_{(2,21)}=3.25$; $p>0.05$, no main effect of time after the CPP test $F_{(1,21)}=1.34$; $p>0.05)$. There was, however, evidence for remodeling, as the total number of MSBs significantly increased in the AMPH CPP group compared with the delayed pairing (Fig. $3 d)(p<0.01)$ and saline controls (Fig. $3 d)(p<0.01)$ and the density of MSBs increased (Table 1) [two-way ANOVA, main effect of behavioral conditioning $F_{(2,21)}=7.96 ; p<0.01$; AMPH CPP versus saline $(1$ h) $p<0.05$; AMPH CPP versus saline $(24 \mathrm{~h}) p<0.05$; AMPH CPP versus delayed pairing $(1 \mathrm{~h}) p<0.05$; AMPH CPP versus delayed pairing $(24 \mathrm{~h}) p<0.05$ ], indicating that synaptic remodeling occurred through growth of additional synaptic sites on existing terminals (i.e., single synaptic boutons becoming multisynaptic). There was no difference in the density of MSBs at $1 \mathrm{~h}$ compared with $24 \mathrm{~h}$ after the CPP test $(p>0.05)$ further verifying the strength of the change.

When we correlated the number and density of excitatory synapses, quantified at $24 \mathrm{~h}$ after the CPP test, with CPP scores (i.e., the time spent in the paired minus the time spent in the unpaired chamber during the CPP test), we found a significant positive correlation between the number and density of asymmetric synapses and the CPP score (Table 2) (number Pearson's $r=0.47 ; p<0.05$; density Pearson's $r=0.46 ; p<0.05$ ). There was also a significant positive correlation in the number and density of asymmetric axospinous synapses (Table 2, number Pearson's $r=$ 0.47; $p<0.05$; density Pearson's $r=0.47 ; p<0.05$ ), and MSBs (Table 2, number Pearson's $r=0.61 ; p<0.01$; density Pearson's $r=$ $0.61 ; p<0.01)$ to CPP score, but not asymmetric axodendritic synapses to CPP score (Table 2, number Pearson's $r=0.14 ; p>0.05$; density Pearson's $r=0.13 ; p>0.05)$, thereby suggesting that new synapse formation onto spines of pyramidal neurons was of primary importance for context-drug associations.

To assess the functional significance of the anatomical findings, we asked whether BLA pyramidal neurons displayed electrophysiological properties that reflected an increase in the number of excitatory contacts. We first examined whether AMPH CPP had an effect on measures of spontaneous synaptic activity. BLA pyramidal neurons displayed frequent spontaneous postsynaptic potentials, usually occurring in groups of spontaneous events or barrages, observed during in vivo intracellular recordings from neurobiotin-filled neurons. In agreement with the anatomical findings, the AMPH CPP group, but not delayedpairing or saline groups, showed an increase in the frequency of spontaneous synaptic events (Fig. 4a) (inter-event interval AMPH CPP $0.54 \pm 0.09$ s, $n=13$ neurons from seven rats; saline $0.92 \pm 0.10 \mathrm{~s}, n=11$ neurons from five rats; delayed pairing $0.89 \pm$ $0.10 \mathrm{~s}, n=11$ neurons from five rats; one-way ANOVA $F_{(2,32)}=$ 7.33; $p<0.01, p_{a}=0.012$; AMPH CPP versus saline $p<0.01, p_{a}$ $<0.05$; AMPH CPP versus delayed pairing $\left.p<0.01, p_{a}<0.05\right)$. There was no difference in the frequency of spontaneous synaptic events between the delayed-pairing and saline control groups $(p>0.05)$. The increase in the frequency of spontaneous events was observed along with an increase in the number of spontaneous synaptic events during barrages of synaptic activity (Fig. 4) (AMPH CPP $8.9 \pm 0.8$ EPSPs/burst, $n=13$ neurons, 7 rats; saline $5.9 \pm 0.8 \mathrm{EPSPs} /$ burst, $n=11$ neurons, 5 rats; delayed pairing $6.4 \pm 0.9 \mathrm{EPSP} /$ burst, $n=11$ neurons, 5 rats; one-way ANOVA $F_{(2,32)}=3.90 ; p<0.05, p_{a}=0.028$; AMPH CPP versus saline $p<$ $0.05, p_{a}<0.05$; AMPH CPP versus delayed pairing $p<0.05, p_{a}<$ $0.05)$. There was no difference in the mean number of EPSPs per burst between the delayed-pairing and saline control groups $(p>0.05)$. Thus, in the AMPH CPP group, both anatomical and physiological measures point to increased synaptic activity onto BLA pyramidal neurons. There was not a significant difference in the frequency of the bursts of activity (AMPH CPP $0.84 \pm 0.16$ $\mathrm{Hz}, n=13$ neurons, 7 rats; saline $0.81 \pm 0.18 \mathrm{~Hz}, n=11$ neurons, 5 rats; delayed pairing $0.77 \pm 0.16 \mathrm{~Hz}, n=11$ neurons, 5 rats; one-way ANOVA $\left.F_{(2,32)}=0.099 ; p>0.05\right)$, as would be expected, since the bursts of activity are tied to the neuronal activity of other regions, which overall is dependent upon anesthesia state, and closely tied to EEG activity. Accompanying the change in the frequency of events was an increase in the average amplitude of spontaneous synaptic events for animals in the AMPH CPP group (Fig. 5a) (AMPH CPP $10.22 \pm 0.60 \mathrm{mV}, n=13$ neurons, 7 rats; saline $7.66 \pm 0.70 \mathrm{mV}, n=11$ neurons, 5 rats; delayed pairing $7.12 \pm 0.60 \mathrm{mV}, n=11$ neurons, 5 rats; one-way ANOVA $F_{(2,32)}=7.26 ; p<0.01, p_{a}<0.01$; AMPH CPP versus saline $p<0.01, p_{a}<0.05$; AMPH CPP versus delayed pairing $p<$ $\left.0.01, p_{a}<0.05\right)$. There was no difference in the average amplitude of spontaneous synaptic events between the delayed-pairing and saline control groups $(p>0.05)$. As an unbiased measure that incorporates both the frequency and amplitude of spontaneous events, the SD from the average membrane potential was also quantified for each neuron and found to be significantly greater in the AMPH CPP group compared with the control groups (Fig. $5 b$ ) (AMPH CPP $4.26 \pm 0.42 \mathrm{mV}, n=15$ neurons, 7 rats; saline $2.12 \pm 0.22 \mathrm{mV}, n=11$ neurons, 5 rats; delayed pairing $2.61 \pm$ $0.33 \mathrm{mV}, n=11$ neurons, 5 rats; one-way $\operatorname{ANOVA~}_{(2,32)}=9.91$; $p<0.001, p_{a}<0.001$; AMPH CPP versus saline $p<0.01, p_{a}<$ 0.05 ; AMPH CPP versus delayed pairing $\left.p<0.01, p_{a}<0.05\right)$. There was no difference in the SD of the average membrane potential between the delayed-pairing and saline control groups $(p>0.05)$. There was no significant difference in the resting membrane potential between groups (AMPH CPP $-74.9 \pm 1.4$ $\mathrm{mV}, n=15$ neurons, 7 rats; saline $-75.4 \pm 1.7 \mathrm{mV}, n=11$ neurons, 5 rats; delayed pairing $-74.8 \pm 1.9 \mathrm{mV}, n=11$ neurons, 5 rats; one-way ANOVA $\left.F_{(2,34)}=0.051 ; p>0.05\right)$ making it 
unlikely that the differences in the synaptic events were due only to the differences in driving force.

To determine whether the increase in synaptic activity specifically reflects increased glutamatergic inputs, a chloride channel blocker (DNDS, $500 \mu \mathrm{M}$ ) was included in the electrode. After 20-30 min, intracellular DNDS application blocked GABAergic components of synaptic inputs evoked by stimulation (Fig. 6). Furthermore, intracellular DNDS application blocked spontaneous IPSPs (measured from -60 to $-55 \mathrm{mV}$ ) (Fig. 6). When DNDS was included in the recording pipette, the frequency of observed spontaneous excitatory synaptic events was significantly greater in pyramidal neurons recorded in AMPH CPP rats compared with the control groups (inter-event interval AMPH CPP $0.56 \pm 0.08 s, n=6$ neurons, 5 rats; saline $1.02 \pm 0.16 \mathrm{~s}, n=6$ neurons, 4 rats; delayed pairing $0.96 \pm$ $0.08 \mathrm{~s}, n=6$ neurons, 4 rats; one-way ANOVA $F_{(2,15)}=4.87 ; p<0.05, p_{a}<$ 0.05; AMPH CPP versus saline $p<0.01$, $p_{a}<0.05 ;$ AMPH CPP versus delayed pairing $\left.p<0.01, p_{a}<0.05\right)$. There was no difference in the inter-event interval observed between neurons in the saline and delayed-pairing groups ( $p>0.05)$. When DNDS was applied in the recording pipette, the SD of the membrane potential was also greater in pyramidal neurons recorded in AMPH CPP rats than in control rats (AMPH CPP $4.15 \pm 0.69 \mathrm{mV}, n=6$ neurons, 5 rats; saline $2.01 \pm 0.17 \mathrm{mV}, n=6$ neurons, 4 rats; delayed pairing $2.48 \pm 0.30$ $\mathrm{mV}, n=6$ neurons, 4 rats; one-way $\operatorname{ANOVA} F_{(2,15)}=6.38 ; p<0.01, p_{a}=0.017$; AMPH CPP versus saline $p<0.01, p_{a}<$ 0.05; AMPH CPP versus delayed pairing $\left.p<0.01, p_{a}<0.05\right)$. No difference was detected in the SD of the membrane potential between neurons with DNDS in the delayed-pairing and saline groups $(p>0.05)$. Because DNDS blocks chloride channels activated by GABAergic transmission, the recorded spontaneous events are likely to be spontaneous EPSPs (sEPSPs) mediated by glutamatergic afferents. Further confirming the excitatory, glutamatergic nature of these events, action potential firing was associated with these sEPSPs when the membrane potential was depolarized with prolonged injection of positive current (Fig. 6). These data indicate that the increased frequency of synaptic events reflects an increase in sEPSPs. The question then arises as to whether this elevation in the frequency of sEPSPs is due to presynaptic changes consistent with an increase in the number of excitatory synapses.

\section{A change in presynaptic function leads to more active synapses}

An increase in the frequency of sEPSPs could result from a "scaling up" of active neuronal networks, not just an increase in synapse number. However, if there is an increase in the number of active synapses, perhaps caused by sprouting of additional axonal branches or increased terminal contacts, we would expect that measures of presynaptic function would be enhanced by AMPH CPP. We examined the efficacy of synaptic inputs from the hippocampal formation (entorhinal cortex/subiculum), a potential source of the synaptic changes after contextual learning. The latencies of the EPSPs evoked by electrical stimulation $(8.3 \pm 0.46$ $\mathrm{ms}$; range 5.4-12.0 ms), along with the monotonic rise of the EPSP, are consistent with a monosynaptic input.

Under control conditions, there was little paired-pulse depression. There was an increase of the paired-pulse ratio after AMPH CPP compared with the control groups (Fig. 5c) (pairedpulse ratio with intracellular DNDS AMPH CPP $1.46 \pm 0.12, n=$ 6 neurons, 5 rats; saline $1.05 \pm 0.06, n=6$ neurons, 4 rats; delayed pairing $1.11 \pm 0.06, n=6$ neurons, 4 rats; one-way ANOVA $F_{(2,15)}=6.73 ; p<0.01, p_{a}=0.014$; AMPH CPP versus saline $p<0.01, p_{a}<0.05$; AMPH CPP versus delayed pairing $p<$ $\left.0.01, p_{a}<0.05\right)$. There was no difference in the paired-pulse ratio between the delayed-pairing and saline groups $(p>$ $0.05)$. An increase of synaptic depression is consistent with a presynaptic change, and has been found to often occur in conditions associated with higher release probabilities (Thomson et al., 1993; Debanne et al., 1996; Thomson and Bannister, 1999; Trommershäuser et al., 2003). This may imply depletion of neu- 
a

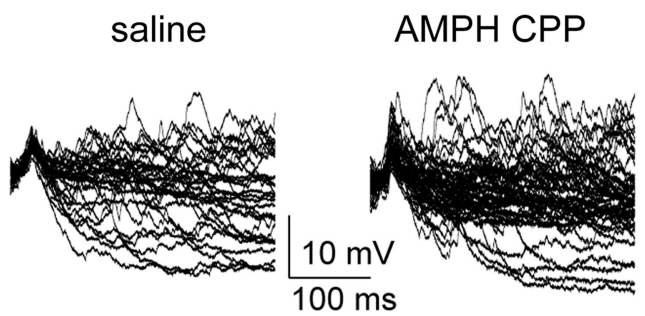

b

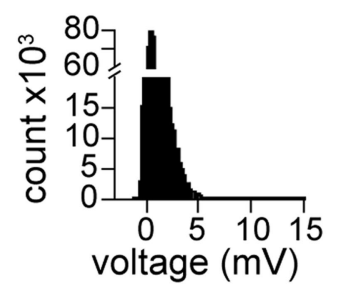

C

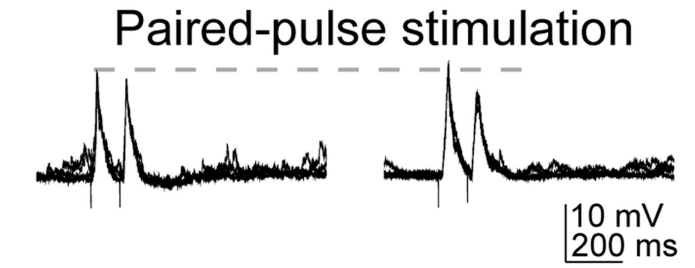

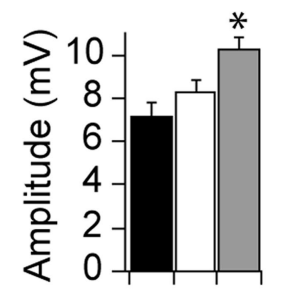
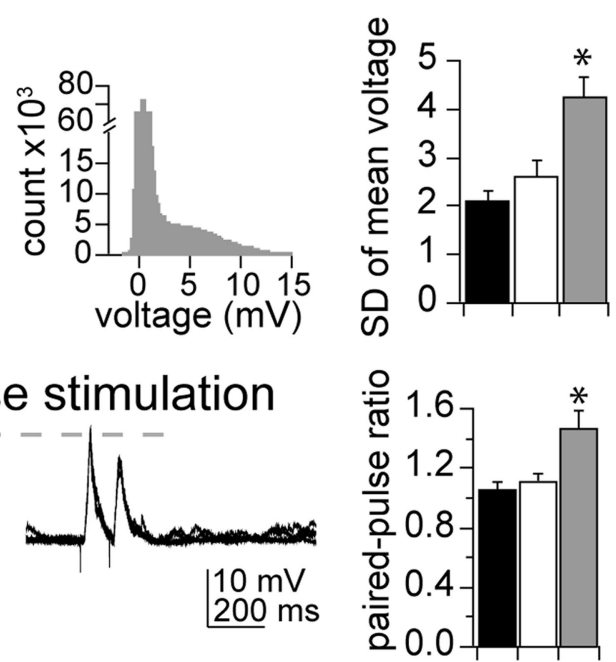

saline delayed pairing

AMPH CPP

Figure 5. AMPH CPP increased other in vivo measures of excitatory synaptic drive. $\boldsymbol{a}$, The amplitude of measured spontaneous EPSPs was increased by AMPH CPP. There was no difference in the amplitude of measured spontaneous EPSPs between neurons of the delayed-pairing and saline groups. Data are expressed as the mean \pm SEM. $\boldsymbol{b}$, To obtain a measure that is less biased by analysis procedures for identifying synaptic events, the fluctuation of the membrane potential was used as an additional index of the frequency and amplitude of spontaneous synaptic events. The SD of the mean membrane potential was increased in the AMPH CPP group (represented graphically as the distribution of the membrane potential). There was no difference between neurons in the delayed-pairing and saline control groups in the SD of the membrane potential. Data are expressed as the mean \pm SEM. $c$, To further provide evidence for a synaptic change after AMPH CPP, the paired-pulse ratio of a stimulated synaptic input was measured. The paired-pulse ratio, in the presence of DNDS, was increased for the AMPH CPP rats, which is consistent with increased synaptic drive. There was no difference in the paired-pulse ratio between the delayed-pairing and saline groups. Data are expressed as the mean \pm SEM. ${ }^{*} p<0.05$ versus the delayed-pairing and saline groups.

rotransmitter by the first pulse, and that a greater proportion of vesicles are released in response to the first pulse (Katz and Miledi, 1968; Charlton et al., 1982; Zucker and Regehr, 2002). While a presynaptic change is the most parsimonious explanation, other factors could contribute. To verify these conclusions, we also examined the CV of EPSP amplitude after stimulation of inputs from the hippocampal formation, an additional index of presynaptic reliability. We found a smaller CV of EPSP amplitude in the AMPH CPP group compared with controls (CV of EPSP amplitude with intracellular DNDS AMPH CPP 1.01 $\pm 0.41, n=$ 6 neurons, 5 rats; saline $1.68 \pm 0.34, n=6$ neurons, 4 rats; delayed pairing $1.52 \pm 0.45, n=6$ neurons, 4 rats; one-way ANOVA $F_{(2,15)}=4.62 ; p<0.05, p_{a}=0.033$; AMPH CPP versus saline $p<0.05, p_{a}<0.05$; AMPH CPP versus delayed pairing $p<$ $\left.0.05, p_{a}<0.05\right)$. There was no difference in the CV of EPSP amplitude between the delayed-pairing and saline groups $(p>$ 0.05). This is further evidence in support of the idea that inputs from the hippocampus are strengthened and may underlie the changes in spontaneous EPSPs observed. The increase in the frequency of sEPSPs combined with a change in the paired-pulse ratio, decreased CV of EPSP amplitude, and increased number of asymmetric synapses is consistent with an increase in the number of functionally active excitatory synapses onto BLA pyramidal neurons after AMPH CPP.

To further link the electrophysiological measures with behavioral indices of context-drug learning, the frequency of spontaneous synaptic events was correlated with the CPP scores. There was a significant positive correlation between the CPP scores and the frequency of spontaneous synaptic events in AMPH CPP rats (Table 1) (Pearson's $r=0.48 ; p<0.05$ ). These data are consistent with an increase in presynaptic drive of BLA pyramidal neurons underlying context-drug learning, a change that likely reflects the large increase in the number of excitatory inputs.

\section{Discussion}

Drug seeking, and the memory required for this behavior, is a powerful drive that leads to further drug taking and relapse after abstinence. In Figure 7, we summarize our findings by illustrating how an increase in BLA excitatory synaptic number and drive could enhance drug-seeking behavior. No studies to date have shown a change in synapse number in the BLA after rewarding or aversive conditioning. Our earlier work showed that AMPH CPP increased the density of synaptophysin, a synaptic protein (Rademacher et al., 2006), but augmented levels of this protein could be accounted for by changes in synaptic vesicles or bouton size. Fear conditioning also produced increases in spinophilin and synaptophysin in the BLA (Radley et al., 2006; Nithianantharajah and Murphy, 2008), but those elevations do not necessarily signal a change in synapse number either. The present results therefore provide novel insights into how BLA circuits adapt with associative learning and show that drug alone is insufficient to change BLA wiring. Nevertheless, the question arises as to whether the exposure to the environmental context paired with drug or the relatively novel environment in which drug was administered elicited the synaptic changes.

Previous studies have shown that repeated psychostimulant administration in the home cage leads to an increase in spines and axospinous contacts (Robinson and Kolb, 2004; Morrow et al., 2007; Morshedi et al., 2009). However, those investigations did not test whether associative learning or novelty/drug interactions underpinned the changes. Environmental novelty has no effect on the absorption, bioavailability, or distribution of AMPH to the brain (Badiani et al., 1997) or AMPH-induced dopamine release (Badiani et al., 1998), but the acute locomotor activating effects of AMPH can be modulated by the context in which it is administered (Badiani et al., 1995a,b; Crombag et al., 1996). Moreover, the locomotor response and the degree of BLA neuronal activation (shown with fos) are greater when AMPH is administered in a novel environment than in the home cage (Badiani et al., 1995a,b, 1998; Ostrander et al., 2003). Such effects are due, at least in part, to contextual cues predictive of AMPH (Badiani et al., 1995a; Crombag et al., 1996). Thus, even though the delayed-pairing group was conditioned in a novel environ- 


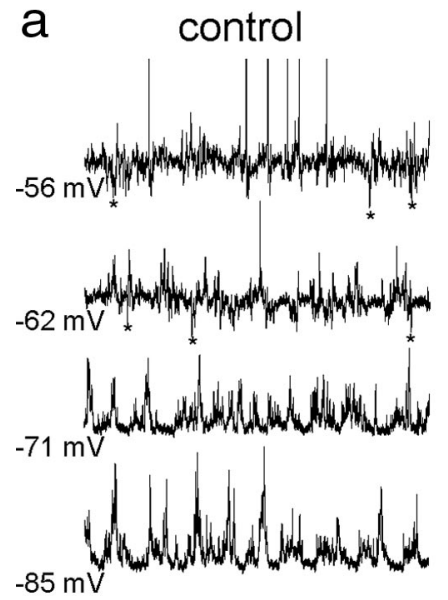

b DNDS $1 \mathrm{~min}$

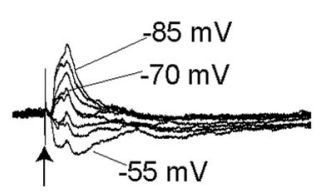

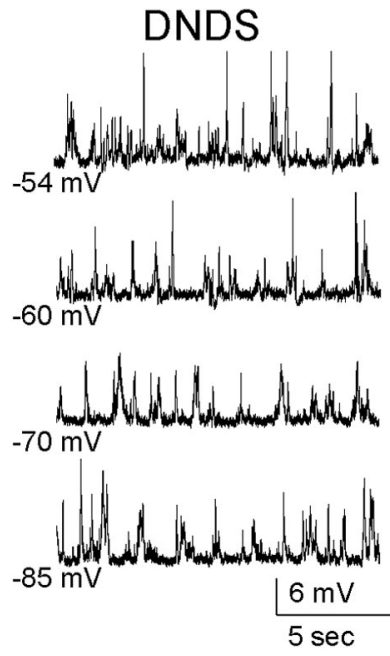

DNDS $20 \mathrm{~min}$

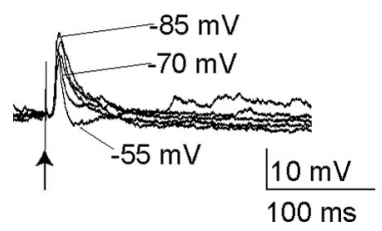

Figure 6. Intracellular blockade of IPSPs with DNDS. $\boldsymbol{a}$, Inclusion of $500 \mu \mathrm{m}$ DNDS in the recording electrode resulted in a decrease in the occurrence of spontaneous hyperpolarizing events (examples of IPSPs at *; baseline frequency $0.83 \pm 0.21 \mathrm{~Hz}$; DNDS frequency $0.03 \pm 0.01 \mathrm{~Hz}$ ), measured between -60 and $-50 \mathrm{mV}$. Also apparent are the action potentials evoked by spontaneous events (action potentials are truncated), indicative of their excitatory nature. $\boldsymbol{b}$, DNDS blocked evoked IPSPs, visible as the downward deflection in these overlaid traces of the response to synaptic stimulation (at arrow) at a range of membrane potentials ( -85 to $-55 \mathrm{mV})$.

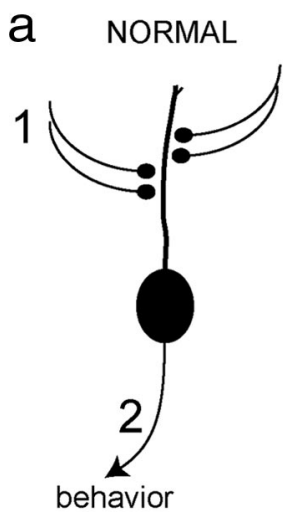

behavior b CONTEXT-DRUG

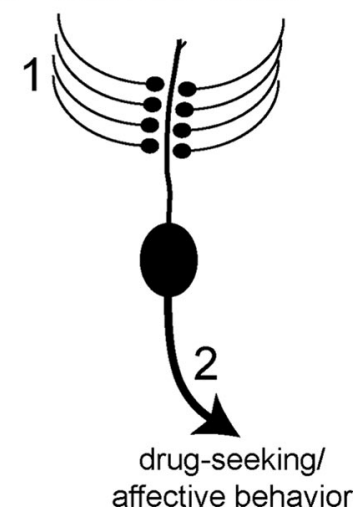

Figure 7. The formation of associations between context and drug modify the activity of the BLA. Neuronal activity in the BLA determines its contribution to behavior. $\boldsymbol{a}$, Synaptic inputs (1) drive the activity of BLA neurons and its contribution to behavior (2). $\boldsymbol{b}$, However, after repeated context-drug associations, there is an increase in the number of excitatory inputs to BLA neurons (1), resulting in increased BLA neuronal activity and drug-seeking behavior (2).

ment that was physically dissimilar to the home cage, and the time they spent in the novel compartment was equal to the time the AMPH CPP group spent in that environment, delayed-pairing rats were injected in the home cage. These data indicate that drug/novelty interactions could have facilitated the associative learning and the synaptic changes in the AMPH CPP group.

In the present study, we found the increase in the number of excitatory synapses and the accompanying facilitation of excitatory connections at $24 \mathrm{~h}$ after the CPP test. It is therefore important to question how the timing of the synaptic measurements affected the changes in excitatory circuits (Kourrich et al., 2007). The timing of our electrophysiological experiments, by their nature, was varied, for there is always a delay while a neuron is being successfully impaled and stabilized in vivo. Since it was important to determine whether the synaptic changes were due to contextual associations during the test or other phenomena related to re-exposure to the CPP apparatus, we also quantified synapses anatomically at $1 \mathrm{~h}$ after the CPP test. We observed no difference in the number or density of excitatory synapses at $1 \mathrm{~h}$ compared with $24 \mathrm{~h}$ after the test, which would indicate that neither the timing of the test nor the withdrawal from drug affects the already formed structural changes in excitatory circuitry brought about by the formation of context-drug associations.

An asymmetric specialization is the morphological hallmark of excitatory, glutamatergic synapses, and the vast majority of spines in the BLA are found on glutamatergic, pyramidal, projection neurons (Brinley-Reed et al., 1995). After AMPH CPP, the frequency of excitatory synaptic events recorded from pyramidal neurons was increased, which could be caused by several factors. While it would be difficult to rule out a change in the global activity of neuronal networks that provide input to the BLA, the most parsimonious explanation, given the anatomical data, is that the total number of active synapses increases and that many of these are newly formed contacts. Furthermore, the paired-pulse data indicate that a presynaptic change (Schulz et al., 1994), independent of the background activity of neuronal networks, contributes to the increase of excitatory events. Because of the potential difficulties with clearly dissociating synaptic events in vivo, a secondary approach of measuring the SD of the mean membrane potential was used. This approach allows quantification of the fluctuation of the membrane potential without assumptions regarding detection of the number of synaptic events or their frequency. These results are also consistent with increased synaptic activity.

The function of the BLA has been associated with fear conditioning. Studies have confirmed an increase in afferent drive after cue-specific fear conditioning (Rogan et al., 1997; Schroeder and Shinnick-Gallagher, 2005) and after cue-specific reward learning (Tye et al., 2008). Our results not only confirm these findings, but also demonstrate that context-dependent reward association facilitates excitatory inputs to the BLA, and provide for the first time an in vivo synaptic basis for that change. While our results cannot prove that all forms of learning lead to a change in excitatory drive, they specifically provide a novel insight into the basis for the associations that underlie drug-related learning.

The increases in synapses and synaptic drive may originate from several sources. Previous studies have demonstrated that the orbital frontal cortex is necessary for BLA neuronal responses to reward-paired cues (Saddoris et al., 2005), implicating this as one source. Inputs from the thalamus or cortex become potentiated after cue-specific reward or aversive learning (McKernan and Shinnick-Gallagher, 1997; Rogan et al., 1997; Schroeder and Shinnick-Gallagher, 2005; Tye et al., 2008), indicating that these brain regions may also be required. Contextual learning, such as CPP, however, relies upon the hippocampus (Olmstead and Franklin, 1997; Ferbinteanu and McDonald, 2001; Shen et al., 2006), and hippocampal inputs to the BLA play a significant role in contextual aspects of amygdala-dependent learning (Maren and Hobin, 2007). Results from our present study indicate that 
excitatory inputs from the hippocampal formation are indeed a likely source of the facilitated drive of BLA neurons.

We favor the interpretation that the increased number of synapses results from the formation of additional excitatory synapses by existing axonal inputs rather than from the growth of new long-range connections. This view is supported by an increase in the number of MSBs after AMPH CPP. A splitting of axonal terminals into multiple inputs to the same neurons will have several ramifications. One consequence would be an increase in the amplitude of EPSPs when a given axon is activated, caused by increased synchronous release at multiple synaptic sites. An additional expected outcome would be an increase in the frequency of spontaneous EPSPs due to the increase in the total number of synaptic connections, coupled with the increased probability of release events in response to an action potential, that results from an increased number of MSBs. An increase in the number of synapses could also increase the capacity of the synaptic connections for plasticity and storage of associative events (Chklovskii et al., 2004; Bruel-Jungerman et al., 2007), potentially serving as a substrate for context-drug associations. From a behavioral standpoint, the increase in the number of active excitatory synapses would mean that the amygdala would be more readily recruited, and contribute to ongoing behavior in response to sets of inputs. In particular, the BLA would drive behavior in response to a set of contextual cues when the rat is tested for CPP.

The mechanisms underlying the ability of environmental context to drive the pursuit of drug are unknown, but our demonstration of increases in the number of excitatory synapses and synaptic activity in the BLA after AMPH CPP provides one explanation for this drive (Fig. 7). Such plasticity, if long-lasting, could be the underlying cause for the indelible nature of drugrelated learning and responsible for the high relapse rates seen when an individual is re-exposed to environments in which the drug had been previously used (O'Brien et al., 1998). This plasticity could be targeted by novel therapies aimed at reversing these negative consequences of drug addiction.

\section{References}

Badiani A, Anagnostaras SG, Robinson TE (1995a) The development of sensitization to the psychomotor stimulant effects of amphetamine is enhanced in a novel environment. Psychopharmacology (Berl) 117:443-452.

Badiani A, Browman KE, Robinson TE (1995b) Influence of novel versus home environments on sensitization to the psychomotor stimulant effects of cocaine and amphetamine. Brain Res 674:291-298.

Badiani A, Camp DM, Robinson TE (1997) Enduring enhancement of amphetamine sensitization by drug-associated environmental stimuli. J Pharmacol Exp Ther 282:787-794.

Badiani A, Oates MM, Day HE, Watson SJ, Akil H, Robinson TE (1998) Amphetamine-induced behavior, dopamine release, and c-fos mRNA expression: modulation by environmental novelty. J Neurosci 18:10579-10593.

Black JE, Isaacs KR, Anderson BJ, Alcantara AA, Greenough WT (1990) Learning causes synaptogenesis, whereas motor activity causes angiogenesis, in cerebellar cortex of adult rats. Proc Natl Acad Sci U S A 87:5568-5572.

Bridges RJ, Worrell RT, Frizzell RA, Benos DJ (1989) Stilbene disulfonate blockade of colonic secretory $\mathrm{Cl}$ - channels in planar lipid bilayers. Am J Physiol 256:C902-C912.

Brinley-Reed M, Mascagni F, McDonald AJ (1995) Synaptology of prefrontal cortical projections to the basolateral amygdala: an electron microscopic study in the rat. Neurosci Lett 202:45-48.

Bruel-Jungerman E, Davis S, Laroche S (2007) Brain plasticity mechanisms and memory: a party of four. Neuroscientist 13:492-505.

Calverley RK, Bedi KS, Jones DG (1988) Estimation of the numerical density of synapses in rat neocortex. Comparison of the 'disector' with an 'unfolding' method. J Neurosci Methods 23:195-205.
Charlton MP, Smith SJ, Zucker RS (1982) Role of presynaptic calcium ions and channels in synaptic facilitation and depression at the squid giant synapse. J Physiol 323:173-193.

Chklovskii DB, Mel BW, Svoboda K (2004) Cortical rewiring and information storage. Nature 431:782-788.

Crombag HS, Badiani A, Robinson TE (1996) Signalled versus unsignalled intravenous amphetamine: large differences in the acute psychomotor response and sensitization. Brain Res 722:227-231.

Day M, Wang Z, Ding J, An X, Ingham CA, Shering AF, Wokosin D, Ilijic E, Sun Z, Sampson AR, Mugnaini E, Deutch AY, Sesack SR, Arbuthnott GW, Surmeier DJ (2006) Selective elimination of glutamatergic synapses on striatopallidal neurons in Parkinson disease models. Nat Neurosci 9:251-259.

Debanne D, Guérineau NC, Gähwiler BH, Thompson SM (1996) Pairedpulse facilitation and depression at unitary synapses in rat hippocampus: quantal fluctuation affects subsequent release. J Physiol 491:163-176.

De Groot DM (1988) Comparison of methods for the estimation of the thickness of ultrathin tissue sections. J Microsc 151:23-42.

de Groot DM, Bierman EP (1986) A critical evaluation of methods for estimating the numerical density of synapses. J Neurosci Methods 18:79-101.

Dudek SM, Friedlander MJ (1996) Intracellular blockade of inhibitory synaptic responses in visual cortical layer IV neurons. J Neurophysiol 75:2167-2173.

Ferbinteanu J, McDonald RJ (2001) Dorsal/ventral hippocampus, fornix, and conditioned place preference. Hippocampus 11:187-200.

Geinisman Y, deToledo-Morrell L, Morrell F (1991) Induction oflong-term potentiation is associated with an increase in the number of axospinous synapses with segmented postsynaptic densities. Brain Res 566:77-88.

Geinisman Y, deToledo-Morrell L, Morrell F, Heller RE, Rossi M, Parshall RF (1993) Structural synaptic correlate of long-term potentiation: formation of axospinous synapses with multiple, completely partitioned transmission zones. Hippocampus 3:435-445.

Honecker H, Coper H (1975) Kinetics and metabolism of amphetamine in the brain of rats of different ages. Nauyn Schmiedebergs Arch Pharmacol 291:111-121.

Hunter A, Stewart MG (1993) Long-term increases in the numerical density of synapses in the chick lobus parofactorius after passive avoidance training. Brain Res 605:251-255.

Ingham CA, Hood SH, Taggart P, Arbuthnott GW (1998) Plasticity of synapses in the rat neostriatum after unilateral lesion of the nigrostriatal dopaminergic pathway. J Neurosci 18:4732-4743.

Katz B, Miledi R (1968) The role of calcium in neuromuscular facilitation. J Physiol 195:481-492.

Kleim JA, Lussnig E, Schwarz ER, Comery TA, Greenough WT (1996) Synaptogenesis and Fos expression in the motor cortex of the adult rat after motor skill learning. J Neurosci 16:4529-4535.

Kleim JA, Vij K, Ballard DH, Greenough WT (1997) Learning-dependent synaptic modifications in the cerebellar cortex of the adult rat persist for at least four weeks. J Neurosci 17:717-721.

Kleim JA, Freeman JH Jr, Bruneau R, Nolan BC, Cooper NR, Zook A, Walters D (2002) Synapse formation is associated with memory storage in the cerebellum. Proc Natl Acad Sci U S A 99:13228-13231.

Knott GW, Holtmaat A, Wilbrecht L, Welker E, Svoboda K (2006) Spine growth precedes synapse formation in the adult neocortex in vivo. Nat Neurosci 9:1117-1124.

Kourrich S, Rothwell PE, Klug JR, Thomas MJ (2007) Cocaine experience controls bidirectional synaptic plasticity in the nucleus accumbens. J Neurosci 27:7921-7928.

Lang EJ, Paré D (1997) Synaptic and synaptically activated intrinsic conductances underlie inhibitory potentials in cat lateral amygdaloid projection neurons in vivo. J Neurophysiol 77:353-363.

Maren S, Hobin JA (2007) Hippocampal regulation of context-dependent neuronal activity in the lateral amygdala. Learn Mem 14:318-324.

McDonald AJ (1982) Neurons of the lateral and basolateral amygdaloid nuclei: a Golgi study in the rat. J Comp Neurol 212:293-312.

McKernan MG, Shinnick-Gallagher P (1997) Fear conditioning induces a lasting potentiation of synaptic currents in vitro. Nature 390:607-611.

Millhouse OE, DeOlmos J (1983) Neuronal configurations in lateral and basolateral amygdala. Neuroscience 10:1269-1300.

Morrow BA, Hajszan T, Leranth C, Elsworth JD, Roth RH (2007) Prenatal exposure to cocaine is associated with increased number of spines synapses in rat prelimbic cortex. Synapse 61:862-865. 
Morshedi MM, Rademacher DJ, Meredith GE (2009) Increased synapses in the medial prefrontal cortex are associated with repeated amphetamine administration. Synapse 63:126-135.

Nithianantharajah J, Murphy M (2008) Auditory specific fear conditioning results in increased levels of synaptophysin in the basolateral amygdala. Neurobiol Learn Mem 90:36-43.

O’Brien CP, Childress AR, Ehrman R, Robbins SJ (1998) Conditioning factors in drug abuse: can they explain compulsion? J Psychopharmacol 12:15-22.

Olmstead MC, Franklin KB (1997) The development of a conditioned place preference to morphine: effects of microinjections into various CNS sites. Behav Neurosci 111:1324-1334.

Ostrander MM, Badiani A, Day HE, Norton CS, Watson SJ, Akil H, Robinson TE (2003) Environmental context and drug history modulate amphetamineinduced c-fos mRNA expression in the basal ganglia, central extended amygdala, and associated limbic forebrain. Neuroscience 120:551-571.

Peters A, Palay SL, Webster HD (1991) The fine structure of the nervous system: neurons and their supporting cells. New York: Oxford UP.

Quirk GJ, Armony JL, LeDoux JE (1997) Fear conditioning enhances different temporal components of tone-evoked spike trains in auditory cortex and lateral amygdala. Neuron 19:613-624.

Rademacher DJ, Kovacs B, Shen F, Napier TC, Meredith GE (2006) The neural substrates of amphetamine conditioned place preference: implications for the formation of conditioned stimulus-reward associations. Eur J Neurosci 24:2089-2097.

Radley JJ, Johnson LR, Janssen WG, Martino J, Lamprecht R, Hof PR, LeDoux JE, Morrison JH (2006) Associative Pavlovian conditioning leads to an increase in spinophilin-immunoreactive dendritic spines in the lateral amygdala. Eur J Neurosci 24:876-884.

Robinson TE, Kolb B (2004) Structural plasticity associated with exposure to drugs of abuse. Neuropharmacology 47:33-46.

Rogan MT, Stäubli UV, LeDoux JE (1997) Fear conditioning induces associative long-term potentiation in the amygdala. Nature 390:604-607.

Rosenkranz JA, Grace AA (2002) Cellular mechanisms of infralimbic and prelimbic prefrontal cortical inhibition and dopaminergic modulation of basolateral amygdala neurons in vivo. J Neurosci 22:324-337.

Rosenkranz JA, Grace AA (2003) Affective conditioning in the basolateral amygdala of anesthetized rats is modulated by dopamine and prefrontal cortical inputs. Ann N Y Acad Sci 985:488-491.

Saddoris MP, Gallagher M, Schoenbaum G (2005) Rapid associative encod- ing in basolateral amygdala depends on connections with orbitofrontal cortex. Neuron 46:321-331.

Schroeder BW, Shinnick-Gallagher P (2005) Fear learning induces persistent facilitation of amygdala synaptic transmission. Eur J Neurosci 22:1775-1783.

Schulz PE, Cook EP, Johnston D (1994) Changes in paired-pulse facilitation suggest presynaptic involvement in long-term potentiation. J Neurosci 14:5325-5337.

Self DW, Nestler EJ (1998) Relapse to drug-seeking: neural and molecular mechanisms. Drug Alcohol Depend 51:49-60.

Shen F, Meredith GE, Napier TC (2006) Amphetamine-induced place preference and conditioned motor sensitization requires activation of tyrosine kinase receptors in the hippocampus. J Neurosci 26:11041-11051.

Sterio DC (1984) The unbiased estimation of number and sizes of arbitrary particles using the disector. J Microsc 134:127-136.

Stuber GD, Klanker M, de Ridder B, Bowers MS, Joosten RN, Feenstra MG, Bonci A (2008) Reward-predictive cues enhance excitatory synaptic strength onto midbrain dopamine neurons. Science 321:1690-1692.

Thomson AM, Bannister AP (1999) Release-independent depression at pyramidal inputs onto specific cell targets: dual recordings in slices of rat cortex. J Physiol 519:57-70.

Thomson AM, Deuchars J, West DC (1993) Large, deep layer pyramidpyramid single axon EPSPs in slices of rat motor cortex display paired pulse and frequency-dependent depression, mediated presynaptically and selffacilitation, mediated postsynaptically. J Neurophysiol 70:2354-2369.

Toni N, Buchs PA, Nikonenko I, Bron CR, Muller D (1999) LTP promotes formation of multiple spine synapses between a single axon terminal and a dendrite. Nature 402:421-425.

Trommershäuser J, Schneggenburger R, Zippelius A, Neher E (2003) Heterogeneous presynaptic release probabilities: functional relevance for short-term plasticity. Biophys J 84:1563-1579.

Tye KM, Stuber GD, de Ridder B, Bonci A, Janak PH (2008) Rapid strengthening of thalamo-amygdala synapses mediates cue-reward learning. Nature 453:1253-1257.

White NM, Carr GD (1985) The conditioned place preference is affected by two independent reinforcement processes. Pharmacol Biochem Behav 23:37-42.

Zucker RS, Regehr WG (2002) Short-term synaptic plasticity. Annu Rev Physiol 64:355-405. 